\title{
Dynamic Analysis into Creeping Reasons of Slowly Moving Hydraulic Cylinder
}

\author{
Fengguo $\mathrm{Li}^{\mathrm{a}}$, Mingfu Yin ${ }^{\mathrm{b}}$ \\ School of mechanical engineering, Tianjin Polytechnic University, Tianjin 300387, China \\ a986821026@qq.com, byinmingfu@163.com
}

Keywords: Load cylinder, Creeping phenomenon, Dynamics modeling, MATLAB simulation.

\begin{abstract}
In this paper, with the opinion of nonlinear dynamics and the triaxial testing machine as the research object, we did the dynamics modeling towards the vertical load cylinder. In the case of taking account of nonlinear friction and nonlinear elastic force, we analyzed the dynamic model of the cylinder, which with low-speed creeping phenomenon, through researching the theory and the matlab simulation. Meanwhile, we made the targeted analysis towards the reason of low-speed creeping for hydraulic cylinder. Calculated the load cylinder when running at low speed, increase the friction force can be effectively restrain the crawling phenomenon and how to guarantee the system stability conclusion.
\end{abstract}

\section{Introduction}

The loading cylinder is used as the actuator in the triaxial test machine, and the pressure can be transformed into mechanical energy by reciprocating rotational motion in a straight line. However, when the loading cylinder is running at low speed, the movement of the stopping time and the fluctuation of the piston are often occurred, which causes the movement of the Pistons to be unsteady, which seriously affects the control precision of the tester. This kind of hydraulic cylinder in the low-speed movement when the jump-type time to move the wave phenomenon, called the creeping phenomenon. Creeping is a very harmful phenomenon, which seriously affects the stability of system work.

Most of the studies have neglected the nonlinearity of spring stiffness, the nonlinearity of friction and the system models, theoretical basis and dynamic characteristics of various nonlinear differential equations. Therefore, this paper mainly analyzes the non-linear viewpoint.

\section{Theoretical Analysis of Low-Speed Crawling of Loaded Oil Cylinder}

The creeping phenomenon of the loading cylinder is essentially a kind of oscillation motion without attenuation. When crawling between the piston and the piston wall of the loading cylinder, the vibration causes should be analyzed qualitatively.

There are many factors that cause the hydraulic cylinder to crawl at low speed: 1 The rigidity of the hydraulic cylinder motion mechanism is too low, forming an elastic system, storing energy and releasing energy to cause intermittent movement; 2 system manufacturing, assembly accuracy is poor, so that friction increases, the force is not good; 3. The working medium in the hydraulic cylinder is mixed with air, and the rigidity of the working medium is reduced to form an elastic body.

According to the above mentioned mechanical and hydraulic reasons are the main reasons for the low-speed crawling of hydraulic cylinders. In the analysis of hydraulic causes, first consider the pressure and flow of hydraulic system, followed by the hydraulic cylinder in the load of the movement mode, and finally the hydraulic cylinder is the main reason for crawling. However, the frictional force, stiffness and damping of the mechanical cause can not be neglected in the oil-cylinder crawling, in order to analyze the reason of creeping more accurately, this paper will expand theoretical research and theoretical verification. 
In order to facilitate the study, the factors that affect the creeping phenomenon are simplified to the influence of friction factor, damping and elastic stiffness.

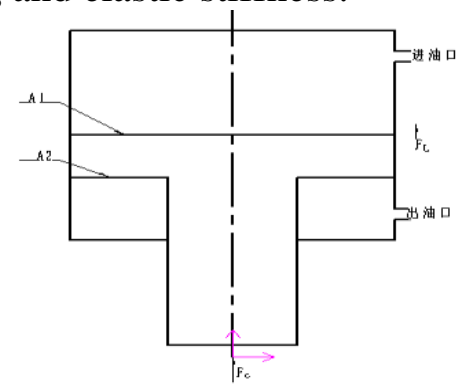

Fig. 1Schematic of loading cylinder

As shown in Fig. 1 above, suppose the inlet pressure, outlet pressure, and the kinetic equation are listed according to Newton's second law:

$$
\mathrm{m} \ddot{x}+F_{\mathrm{c}}+F_{f}+F_{s}=P_{1} A_{1}-P_{2} A_{2}-F_{L}
$$

Medium: M-Piston quality; $F_{\mathrm{C}}$-Viscous resistance; $F_{S}$ - Elastic force; $F_{\mathrm{f}}$ - Friction; $F_{L}$-Load; $P_{1}$ Injection Chamber Pressure ; $P_{2}$ - Back pressure of oil chamber; $A_{1}$ - Stemless Cavity Piston Area ; $A_{2}-$ Area with dry cavity piston.

Order $: \mathrm{y}=x-v_{o} t$, Taylor expansion is carried out in the vicinity of the working point of the nonlinear elastic force and the nonlinear friction forces, and the generation (1) can be:

$$
\begin{gathered}
m \ddot{y}+c \dot{y}+\left(c^{\prime} \dot{y}+c^{\prime \prime} \dot{y}^{2}+c^{\prime \prime \prime} \dot{y}^{3}\right)+\left(k^{\prime} y+\right. \\
\left.k^{\prime \prime} y^{2}+k^{\prime \prime \prime} y^{3}\right)=P_{1} A_{1}-P_{2} A_{2}-F_{L}-F_{f}\left(V_{o}\right) \\
k=\left(v_{o} t\right) ; k^{\prime}=\frac{d k\left(v_{0} t\right)}{d y} ; k^{\prime \prime}=\frac{1}{2} \frac{d^{2} k\left(v_{0} t\right)}{d y^{2}}, c^{\prime}=\frac{d F_{f}\left(v_{o}\right)}{d \dot{y}} ; c^{\prime \prime}=\frac{1}{2} \frac{d^{2} F_{f}\left(v_{o}\right)}{d \dot{y}^{2}} ; \\
c^{\prime \prime \prime}=\frac{1}{3} \frac{d^{3} F_{f}\left(v_{o}\right)}{d \dot{y}^{3}} ;
\end{gathered}
$$

In the formula: $\mathrm{y}, \dot{\mathrm{y}}$ and $\ddot{\mathrm{y}}$ respectively indicate the vibration displacement, velocity and acceleration in the vicinity of the work, and $\mathrm{C}$ is the viscous damping coefficient of the hydraulic cylinder;

$c^{\prime}, c^{\prime \prime}$ and $c^{\prime \prime \prime}$ are the Taylor coefficients of the nonlinear friction in the vicinity of the working point, and $\mathrm{k}, k^{\prime}$ and $k^{\prime \prime} 6$ are the Taylor coefficients of the nonlinear elastic force unfolding near the working point, $F_{f}\left(v_{o}\right)$ is the friction at the working point, and $v_{o}$ is the average running speed of the hydraulic cylinder.

The current working principle of the loading cylinder and the pressure valve determines that the pressure of the hydraulic oil of the inlet and out hydraulic cylinder has micro-fluctuation, the basic obeys the law of simple harmonic oscillation, and can be approximately expressed as $F_{1} \cos \omega t$, which is the excitation source of the system. Therefore, the equation of the nonlinear elastic force can be expressed as:

$$
m \ddot{y}+c_{L} \dot{y}+k_{1} \mathrm{y}+k_{3} y^{3}=F \cos \omega t
$$

In the formula: $c_{L}$ is the damping coefficient, $\mathrm{K}$ is the spring stiffness, and $\omega$ is the vibration frequency.

The amplitude-frequency characteristic curve of forced vibration is drawn by MATLAB: 


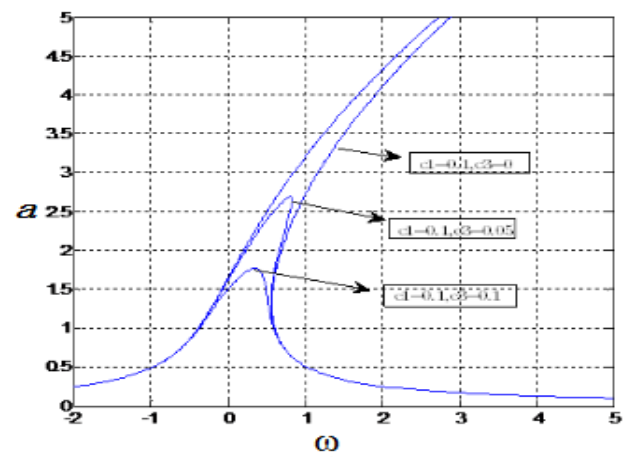

Fig. 2 The amplitude-frequency characteristic curve with damping

As shown in Fig. 2, the quantitative (Control-variable) analysis of the effect of the magnitude of the frequency $\omega$ on the nonlinear Spring Litel expansion. At this time, with the increase of $\omega$, the amplitude also becomes larger, at this time jump phenomenon is more obvious, damping is larger.

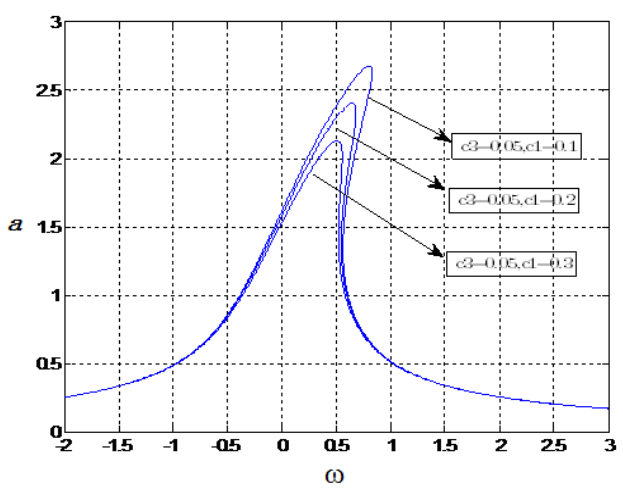

Fig.3 Curve of amplitude-frequency characteristic with damping

As shown in Fig.3,the effect of $\mathrm{C} 1$ on amplitude-frequency C1characteristics is smaller than that of $\mathrm{C} 1$. However, C1items cannot be neglected when analyzing the effect of nonlinear friction on creeping phenomenon.

\section{The Theory Verification of Low-Speed Crawling of Loaded Oil Cylinder}

Through theoretical verification and stability judgement of low speed creeping phenomenon of oil cylinder, we can know that there is no creeping phenomenon of oil cylinder, and now through different frequency changes, the relationship between amplitude and frequency of the system is verified by MATLAB simulation, and the stability of low speed creeping phenomenon of cylinder is proved.

Taking different excitation frequencies, the whole system (6) is simulated, and the time domain waveform and phase diagram of the whole system are obtained to verify the correctness of the influence of the parameters on the amplitude-frequency characteristic curve.

Suppose $\omega=0.5$, as shown in Fig 4:
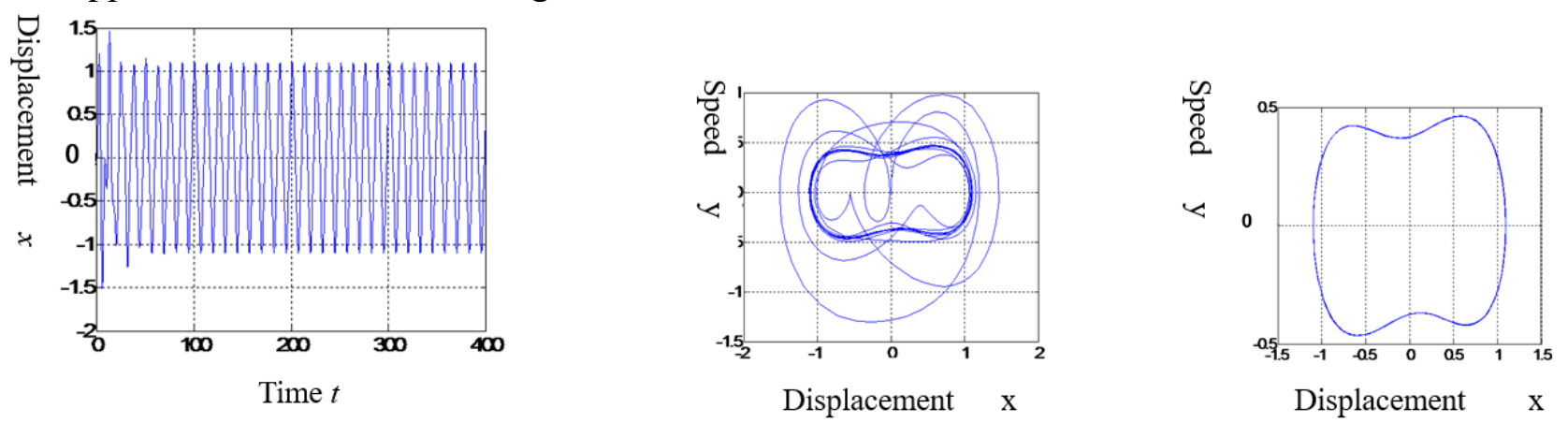

Fig. 4 State Temporal Waveform map

When the two friction surfaces are in the boundary lubrication and some fluid lubrication, as shown in (2) the system is in an unstable state, when the two friction surfaces are in the fluid 
lubrication phase, and the FIG (3) system is in a stable phase. When the loading cylinder is in the crawling motion, the amplitude of the system and amplitude-frequency characteristic curve are approximately equal, and the correctness of the theory is illustrated by fig (1). The same conclusion is obtained when $\Omega$ takes the following values, which further illustrates the correctness of the theory.

Suppose $\omega=1.5$, as shown in Fig5:
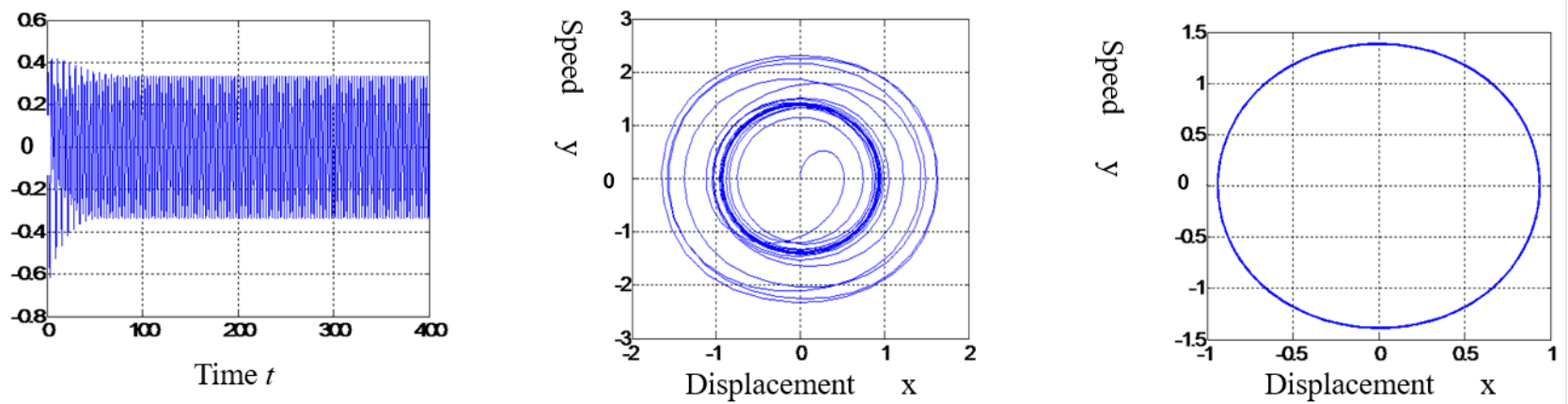

Fig.5 Status Two o'clock field waveform map

The amplitude-frequency characteristic curve of MATLAB simulation 2 shows that when $C 1=0.1$ o'clock, the amplitude fluctuation of the $\mathrm{C} 3=0.1$ waveform is the least stable. As shown in Figure 3 , when $\mathrm{C} 3=0.05$ o'clock, the amplitude fluctuation of the assignment $\mathrm{C} 1=0.3$ waveform is the least stable. By verifying the parameters of the amplitude-frequency characteristic curve, the consistency of the system amplitude and amplitude-frequency characteristic curve is obtained, and the stability of the low speed creeping phenomenon of the loading cylinder is obtained.

\section{Summary}

(1) According to Newton's second theorem, the dynamic modeling of the whole simplified model is carried out, and the average method is used to solve the vibration differential equation without neglecting the nonlinear friction and the nonlinear elastic force, and the amplitude-frequency characteristic curve is drawn. It can be concluded that when the loading cylinder runs at low speed, the increase of friction can effectively restrain the creeping phenomenon of the loading cylinder.

(2) From the theory analysis of the low speed creeping of the oil cylinder, the system stability discrimination to the theory verification, and obtains the system amplitude and the amplitude frequency characteristic curve consistency through the computer simulation form and obtains that when the condition satisfies the characteristic solution formula, the amplitude change of the system is stable namely no creeping phenomenon.

\section{References}

[1]. C. D. Rakopoulos, E. G. Giakoumis, D. T. Hountalas. Experimental and simulation analysis of the transient operation of turbocharged multi - cylinder IDI diesel engine[J]. Int. J. Energy Res.,1998, 224.

[2]. You Liu, Zhi-guo Yuan, Li-yun Fan, Bin-qi Tian. Dynamic characteristic investigation on the fuel pressure of diesel engines electronic in-line pump system[J]. Journal of Marine Science and Application, 2010, 94.

[3]. Wojciech Sochacki. Modelling and analysis of damped vibration in hydraulic cylinder[J]. Mathematical and Computer Modelling of Dynamical Systems, 2015, 211.

[4]. Qiwei Lai, Liang Liang, Jing Li,Shijing Wu, Jun Liu,Jian Guo Zhou. Modeling and Analysis on Cushion Characteristics of Fast and High-Flow-Rate Hydraulic Cylinder[J]. Mathematical Problems in Engineering, 2016.

[5]. Wang Xiujun, Yin Lisong, Fan Hua zhi, Li Yongxi, Ted. Study on mathematical model of hydraulic cylinder creeping phenomenon [J]. Hydraulic and pneumatic, 2013,09:87-89. 
[6]. Wang Linhong, Guo Junjie, Vu, Yang Shuzi. A new study on the reason of low speed creep of hydraulic cylinder [J]. Hydraulic and pneumatic, 2006,09:14-18. 